\title{
Bert Hoffmann
}

\section{Kuba: Die Reform von innen, die nicht stattfand}

Die Renaissance der kubanischen Sozialwissenschaften und die Reaktion der

Staatsführung - eine Sichtung neuerer Publikationen aus K uba

Was Macht ist, hatte Karl W. Deutsch einmal auf bemerkenswerte Weise definiert: »Power is the ability to afford not to learn." In diesem Sinne stellte die kubanische KP im letzten Frühjahr eindrucksvoll unter Beweis, daß sie auch im siebten Jahre nach dem Fall der Berliner Mauer noch i mmer die Macht auf der Karibikinsel hat. Die Macht, und keinen Lernbedarf: Die Parteiführung lancierte einen mit massiven Drohungen vorgetragenen »ideologischen Feldzug«, der die kubanischen Sozialwissenschaftler und Ökonomen, die sich über die Krise des Landes zu eigenständige Gedanken gemacht hatten, wieder in ihre Schranken verweisen sollte. Raúl Castro, seines Zeichens Armee-Chef, Vizepräsident und Bruder Fidels, verlas vor dem Zentralkomitee der kubanischen KP einen zu trauriger Berühmtheit gelangten Bericht des Politbüros: Die Gefahr von Konterrevolution und Imperialismus lauere vor allem im Inneren, bei den Abweichlern in den e igenen Reihen - namentlich und allen voran in den sozialwissenschaftlichen Studienzentren auf der Insel. Hier drohe die Gefahr von »Subversion« und »Diversionismus« und »das Entstehen einer Fünften Kolonne« des Feindes, das es mit aller Entschiedenheit zu bekämpfen gelte (R. Castro 1996, 6f; alle Übersetzungen: B.H.). Mit diesem Frontalangriff stutzte die Staatsfü hrung einem Aufbruch in Kubas akademischer Welt die Flügel, in dem es offener als je zuvor zu einer kontroversen Debatte über die Probleme und Zukunftsperspektiven des kubanischen Sozialismus gekommen war. Diese Debatte nachzuzeichnen und - ein Jahr nach dem Paukenschlag von oben eine vorläufige Bilanz dieses Konflikts zu ziehen, ist Anliegen dieses Be itrags.

Hintergnund der sozialwissenschaftlichen Diskussion in Kuba ist die for tgesetzte und tiefgreifende Krise der sozialistischen Karibikinsel. Zwar ist die deprimierende Untergangsstimmung von vor drei Jahren überwunden, als Zehntausende von Kubanern und Kubanerinnen die Insel auf halsbr e- 
cherischen Flößen verließen. Einige zentrale makroökonomische Indikatoren zeigen sogar erste Signale einer Stabilisierung der Volkswirtschaft (vgl. Hoffmann 1996a, 134ff). Doch die bisherigen Erholungstendenzen sind nicht nur prekär und von Rückschlägen bedroht, sondern es ist auch eine Stabilisierung auf einem denkbar niedrigen Niveau. Zudem geht sie einher mit dem Entstehen einer neuen sozialen Ungleichheit, die von den Kub anern vielfach als sehr kraß empfunden wird. Für praktisch jeden Kubaner und jede Kubanerin hat der so tiefe Sturz der kubanischen Wirtschaft seit dem Zusammenbruch der Verbündeten in Übersee die Lebens perspektiven hinfällig werden lassen, auf die sie nach drei Jahrzehnten Socialismo $\mathrm{Cu}$ bano mit der Sowjetunion im Rücken gebaut hatten. Der Zerfall der ök onomischen Basis hat auch all jene sozialen Netze und Institutionen, die mit der Revolution entstanden waren, einem tiefgreifenden Erosionsproze $\beta$ unterworfen - und neuen zur Geltung verholfen (Hoffmann 1996b). Das politische System selbst ist von diesem Erosionsprozeß keineswegs ausg enommen. In dieser Situation gewinnt die Debatte, die sich in Kubas Soz ialwissenschaften in den letzten drei, vier Jahren entwickelt hat, besondere Bedeutung - umso mehr da derartige Diskussionen in anderen öffentlichen Räumen, etwa der Presse oder dem Parlament, gar nicht oder in noch sehr viel eingeschränkterem Maße geführt werden.

Im folgenden soll diese Renaissance der kubanischen Sozialwissenschaften anhand einer Rezension zentraler Publikationen der letzten Jahre skizziert werden. Der erste Teil widmet sich dabei ausführlich einer Arbeit, die den unbestrittenen Mittelpunkt der Diskussion über die wirtschaftlichen R eformperspektiven darstellt. Im zweiten Teil soll dann ein Überblick über eine Reihe von Veröffentlichungen gegeben werden, die sich bei der An alyse der politischen und gesellschaftlichen Krise auf Neuland begaben.

\section{Eine sozialistische Reformalternative gegen die Dollarisierung der Wirtschaft}

Als das Buch Cuba: La restructuración de la economía - Una propuesta para el debate (Kuba: Der Umbau der Wirtschaft - ein Vorschlag für die Debatte) in einem staatlichen kubanischen Verlag erschien, war dies eine mittlere Sensation für die akademische Welt der Insel. Noch am gleichen Abend, an dem der Band öffentlich präsentiert wurde, war die gesamte für den Verkauf in Pesos vorgesehene Auflage bereits vergriffen. Denn deutl icher als jede andere Publikation zuvor zeigte die Arbeit von Julio Carranza Valdés, dem Vize-Direktor des Zentrums für Amerika-Studien (CEA), und den beiden CEA-Mitarbeitern Luis Gutiérrez Urdaneta und Pedro Monreal González (Carranza et al. 1995), auf welch hohem wissenschaftlichen $\mathrm{N}$ iveau und mit welchem politischen Selbstbewußtsein in Kuba über die Krise 
des Landes und, mehr noch, über mögliche Alternativen zur aktuellen Pol itik der Regierung geschrieben werden konnte. Das ganze hatte nur einen Haken: die Veröffentlichung des Buches gab nicht den Anstoß zu einer breiten Debatte, wie es die Absicht der Autoren war, sondern markiert im Rückblick bereits den Höhepunkt jenes »akademischen Frühlings«, den das Politbüro in der Folge zur unerwünschten Jahreszeit erklärte.

Schon der Titel signalisiert, auf welcher Ebene das Anliegen von Carranza et al. liegt: Erstens beschäftigt sich das Buch nicht mit einzelnen Aspekten der Krise oder mit ausgewählten Sektoren der Ökonomie, sondern es geht und daran hatte sich bis dahin niemand herangewagt - um ein umfassendes und kohärentes Programm für den »Umbau der Wirtschaft«. Zudem weist der Untertitel darauf hin, daß es sich bei diesem Projekt eben nicht um die Ausformulierung der offiziellen Regierungspolitik handelt, sondern um e inen eigenen »Vorschlag für die Debatte«, um »eine gangbare sozialistische Alternative der Reform des Wirtschaftssystems « (S. 4). Die Ausformuli erung dieser Alternative stellt ein Fundament der gegenwärtigen Politik der Regierung Castro in Frage: daß ihre Politik die einzig mögliche ist. Mehr noch. Mit der Publikation von Carranza et al. wird offensichtlich, daß die Regierung selbst nicht in auch nur ansatzweise ähnlich umfassender Form dargelegt hat, wie denn ihre Pläne für die Zukunft der kubanischen Wir tschaft über das tagesaktuelle Stückwerk hinaus aussehen.

Dem eigentlichen Konzept zum »Umbau der Wirtschaft《 vorgeschaltet haben die Autoren eine Bestandsaufnahme der gegenwärtigen Situation. Di ese führt jene vergleichsweise ungeschminkte Darstellung der Probleme fort, mit der Julio Carranza schon zwei Jahre zuvor mit seinem vielbeachteten Aufsatz Cuba: Los retos de la economía (Kuba: Die Herausforderungen, vor denen die Wirtschaft steht) neue Zeiten in Kubas akademischer Welt einläutete. Carranza hatte darin unter anderem den Beginn der gegenwärt igen Krise nicht auf 1989/90 und die Auflösung von UdSSR und RGW d atiert, sondern bereits auf 1986. Denn schon 1986, so rechnete Carranza vor, zeichneten sich in der Binnenökonomie unübersehbar die Grenzen des bi sherigen, extensiven Wachstumsmodells ab. Auch in Kubas Außenbezi ehungen kam es bereits 1986 zum Bruch, als die Insel ihre stark angewac hsenen Devisenschulden nicht mehr bedienen konnte, den Schuldendienst einseitig aufkündigte und damit ihre internationale Kreditwürdigkeit verlor. "Aus diesen Gründen beschließt die kubanische Regierung 1986, die Wir tschaftsbeziehungen mit den kapitalistischen Ländern auf ein Minimum zu reduzieren und sie stattdessen auf die Länder des RGW, vor allem auf die UdSSR, zu konzentrieren « (Carranza 1992, 133). Es war nicht zuletzt diese politische Entscheidung 1986 - just zu dem Zeitpunkt, als Gorbatschows Perestroika bereits begonnen hatte! -, die den Zusammenbruch der sozial istischen Verbündeten für Kuba so verhängsnisvoll machen sollte. Daran 
anknüpfend fassen Carranza et al. in der Einleitung zu ihrem neuen Buch die Problemlage der kubanischen Wirtschaft in den folgenden drei Punkten zusammen:

»1990 brechen in abrupter Form die bisherigen internationalen Verbindungen der kubanischen Wirtschaft weg. Das Land sieht sich dem Weltmarkt ausgesetzt, die nordamerikanische Blo kkade wird effektiver wirksam (und zusätzlich verschärft durch das Torricelli-Gesetz), und die bis dahin ungelösten Probleme der wirtschaftlichen Effizienz [in der kubanischen Ökonomie] werden offensichtlicher und kostspieliger« (S. 2f).

Zwischen den Zeilen klingt hier schon an, wo die Autoren Handlungsb edarf für die kubanische Politik sehen. Denn die ersten beiden Punkte - den Weltmarkt und die Politik der USA - zu ändern, steht kaum in der Macht der Regierung in Havanna. Zumal die Regierung Castro nach 1990 im Rahmen des Möglichen auf diese beiden Punkte durchaus mit Erfolg re agierte: Sie hat der Blockade-Politik der USA getrotzt; und sie hat die e rzwungene Neuintegration in die Weltwirtschaft aktiv betrieben, den A ußenhandel umgestellt und eine begrenzte Außenöffnung der kubanischen Ökonomie zugelassen. Was mit dem Ausbau des internationalen Tourismus sowie der Förderung von Joint-venture-Unternehmen mit Auslandskapital begann, führte spätestens mit der Legalisierung des US-Dollars im Juli 1993 zu einer regelrechten Zweiteilung der kubanischen Ökonomie in eine Dollar- und eine Peso-Sphäre. In dem eklatanten Auseinanderklaffen dieser beiden Währungswelten rächt sich nun aber, daß eine Antwort auf den dritten Punkt verweigert wurde: Im Sommer 1994 war der durchschnittl iche staatliche Monats(!)lohn, schwarz getauscht, gerade noch anderthalb US-Dollar wert. War der Erhalt des politischen Status quo das allem übe rgeordnete Primat der Wirtschaftspolitik, so war der Niedergang der PesoÖkonomie ihre nach unten offene Variable.

Ein derartig krasses monetäres Mißverhältnis führt fast zwangsläufig zu s ozialen Spannungen sowie zu schwerwiegenden ökonomischen Folgepr oblemen. Wo ein Kellner in einem Touristenrestaurant an drei Abenden a 1lein durch das Trinkgeld soviel verdient wie ein Arbeiter in einem Stah 1werk mit 44-Stunden-Woche im ganzen Jahr, ist es nicht nur schwer, die egalitären Prinzipien der Revolution hochzuhalten, sondern auch die A rbeitsmotivation des Stahlarbeiters. So benennen Carranza et al. die negativen Auswirkungen der fast völligen Entwertung der Löhne: flächende kkend gesunkene Arbeitsdisziplin, temporärer und dauerhafter Absentismus, zunehmende Verlagerung des Gelderwerbs auf den Schwarzmarkt etc.

»Wo der Lohn - die materielle und soziale Anerkennung der Arbeit - nicht mehr das grundl egende Mittel zum Erhalt des persönlichen und familiären Wohlergehens darstellte, hatte all dies sehr negative Auswirkungen auf eine Gesellschaft von Arbeitern - und zwar nicht nur wirtschaftilich, sondern auch ideologisch« (S. 30f).

Der zentrale Ausgangspunkt des Reformprogramms von Carranza et al. ist daher eine Währungsreform. Gerade um den sozialen Gehalt der Revolut i- 
on zu retten, bestehen die Autoren auf der Notwendigkeit, diese unfriedl iche Koexistenz von exklusiven Dollarsphären und darniederliegender Peso-Ökonomie zugunsten einer einheitlichen nationalen Währung aufzuh eben. Damit jedoch liegen die Autoren überkreuz mit der Politik der Regi erung, die sich im Sommer 1994 gegen die Durchführung einer Währung sreform entschied und stattdessen auf die schrittweise Dollarisierung der Ökonomie setzt: Flächendeckend werden im ganzen Land staatliche Dollar-Supermärkte eingerichtet, neue Tankstellen mit digitalen Zapfsäulen und Dollar-Preisen schießen aus dem Boden, und neue staatliche FastFood-Ketten namens »El Rápido« und »Burgui« versorgen die Bevölkerung, soweit sie über Kaufkraft in harter Währung verfügt, mit Hamburgern für zwei Dollar und Pepsi-Büchsen oder Heineken-Bier für einen. Auch für das Problem der Arbeitsmotivation setzt die Regierung auf den Dollar: In den »prioritären « Sektoren, d. h. vor allem in der Exportwirtschaft, erhalten die Arbeiter Prämienanreize in harter Währung (üblich sind z.B. in der T abakproduktion zwischen zwei und 20 Dollar im Monat - vgl. Hoffmann 1996a, 113ff).

An dieser Stelle ist ein Hinweis auf die Entstehung der Studie von Carranza et al. nötig. Das Buch ist die erweiterte und aktualisierte Fassung eines Entwurfs, den die Autoren im April 1994 den für die Wirtschaft Veran twortlichen in der kubanischen Regierung übergaben (selbstverständlich nicht-öffentlich). Das Datum ist von Bedeutung: Das Projekt von Carranza $\&$ co war damit eine reale Handlungsalternative zu jenem Programm der »Sanierung der Staatsfinanzen« ohne vorherige Währungsreform, das die Regierung im Mai 1994 in Gang setzte. Zur Erinnerung sei hinzugefügt, daß das von Carranza et al. skizzierte Reformprojekt damit noch vor den spektakulären Ereignissen des Sommers 1994 entstand, vor den Unruhen in Havanna am 5. August und der folgenden Massenflucht von Zehntause nden auf primitivsten Flößen. Erst auf diesen offenen Ausbruch der bis d ahin latenten sozialen Krise antwortete die Regierung wenige Wochen sp äter mit der Zulassung von Bauern- und Handwerksmärkten. Damit un ternahm sie den bislang wichtigsten Schritt in der Transformation der Funk tionsmechanismen der kubanischen Ökonomie nicht als Teil einer gepla nten Reform, sondern als ein kurzfristig motiviertes Krisenmanagement.

Es ist diese Kurzfristigkeit und Planlosigkeit der staatlichen Politik, die Carranza et al. kritisieren, wenn sie immer wieder die Notwendigkeit der Kohärenz und einer sinnvollen Abfolge der einzelnen wirtschaftspolit ischen Maßnahmen betonen. Auch Carranza et al, hatten die Wiedereröffnung der Bauernmärkte als zentrale Reformmaßnahme gefordert, um die kritisch gewordene Lebensmittelversorgung zu verbessern und um dem ausgeuferten Schwarzmarkt eine legale Alternative entgegenzustellen. $\mathrm{Zu-}$ vor jedoch hätte ein Währungsschnitt, so ihr Argument, den aufgestauten 
Peso-Überhang abschöpfen und zumindest eine relative Gleichheit als Ausgangsbasis schaffen müssen, bevor neue, an den Kräften des Marktes und des Geldes orientierte Verteilungsmechanismen Einzug halten.

Der aufgestaute Geldüberhang, dem in der rationierten Peso-Ökonomie keine legal käuflichen Waren gegenüberstehen, beträgt nach Angaben der Regierung nicht weniger als 14 komplette Monatsgehälter pro erwachsenen Kubaner. Dahinter aber steckt ein noch größeres Problem, denn dieser Überhang ist keineswegs gleichmäßig verteilt. Gerade durch die enormen Preis- und Lohnverzerrungen hatte Kubas Geldvermögen bereits vor (!) der Zulassung legaler Marktmechanismen eine Konzentration lateinamerikan ischen Zuschnitts angenommen. Ein wenig bekannter Aufsatz des Zentrums zum Studium der kubanischen Wirtschaft (CEEC) in Havanna nennt dafür offizielle kubanische Zahlen: »[Im Mai 1994] kontrollierten 15\% der B evölkerung $70 \%$ der umlaufenden Geldmenge [in Pesos]. Bei der auf Bankkonten deponierten Geldmenge verfügten $6 \%$ der Sparer über $50 \%$ der Einlagen (...) [Zudem] ist der Teil der Bevölkerung, der den bedeutsamen Anteil der umlaufenden Geldmenge [in Pesos] auf sich vereinigt, gleic hzeitig auch der, der den größten Teil der Geldmenge in US-Dollars ko ntrolliert « (Marquetti 1995, 19). Seitdem ist die Schere weiter auseinande rgegangen. Damit aber ist die Frage einer Währungsreform keineswegs eine bloß »technische Frage« für den Streit der Experten, sondern von grundl egender und langfristiger Bedeutung: Sie definiert die ökonomischen und sozialen Fundamente jenes neuen Kubas, dessen Entstehung wir derzeit beiwohnen. Ohne diesen Schritt war die materielle Chancengleichheit der kubanischen Gesellschaft bereits verloren, noch bevor die ersten Marktr eformen durchgeführt wurden.

Noch wird in Kuba die Dramatik dieser Geldkonzentration durch die staa tlichen Subventionen und die verbliebenen Reste der Warenverteilung über Rationierungskarten abgefedert. Die Frage für die Zukunft ist jedoch wohl weniger ob, sondern nur in welchem Tempo sowohl Subventionen wie auch rationierte Produkte in den kommenden Jahren reduziert werden.

Das Währungsreform-Argument von Carranza et al. relativiert auch einen der größten Erfolge der Regierungspolitik, die relative Stabilisierung des Peso. Durch das harte staatliche Sparprogramm seit Mai 1994 einerseits und die Ausweitung des Produktangebots andererseits ist es der Regierung gelungen, den Wertverlust des kubanischen Peso zu stoppen und ihm wieder realen Wert zu geben. Lag der schwarze bzw. freie Kurs zwischen Peso und Dollar im Sommer 1994 bei 150:1, so pendelte er zweieinhalb Jahre später nur noch um 25:1. In absoluten Zahlen ergibt dies immer noch einen durchschnittlichen Monatslohn von gerade 7 US-Dollar, doch in der Te ndenz ist der Erfolg durchaus respektabel. Hält man sich aber die ungleiche Verteilung des Peso-Vermögens im Mai 1994 vor Augen, dann war es hi n- 
sichtlich der sozialen Gerechtigkeit fatal, daß die Regierung die Stabilisi erung des Peso ohne einen vorangehenden Währungsschnitt begann; die Stärkung des Peso hat damit weniger den Lohnempfängern genützt als vielmehr den Besitzern der großen Peso-Vermögen. Es ist die bittere Lekt ion von Carranza et al., daß diese Rückkehr der Ungleichheit keineswegs die unvermeidliche Konsequenz externer Zwänge war, sondern Folge einer konkreten Politik der kubanischen Regierung, zu der es durchaus Alternat iven gegeben hätte.

Die Diskussion um die Währungsreform ist nur ein Punkt - wenn auch ein zentraler - in dem Reformprojekt, dessen Blaupause Carranza et al. zeichnen. Sie legen dabei das Schwergewicht ihrer Arbeit auf die grundsätzliche "Architektur« des Umbaus, weniger auf eine detaillierte Bearbeitung der konkreten Einzelfragen, die sich dabei ergeben (was im Rahmen eines 200Seiten-Buches auch nicht zu leisten wäre). Gerade für die konzeptionelle Ausarbeitung machen sich die Autoren die Auseinandersetzung mit der i nternationalen Fachliteratur - ob nun Przeworskis Studien über Reformen in Osteuropa und Lateinamerika oder Kornais Analyse der "s soft budget constraints « in real-sozialistischen Ökonomien - mit einer Selbstverständlic hkeit zu Nutze, die in Kubas Sozialwissenschaften ihresgleichen sucht. Um allen Mißverständnissen vorzubeugen: den Autoren geht es immer um ein ausdrücklich sozialistisches Reformprojekt, nicht um einen »Übergang zum Kapitalismus « (das Buch widmet ein eigenes Kapitel der Kritik an verschiedenen in den USA entwickelten » Transitionsszenarien « für Kuba). Carranza et al. gelingt es dabei, ihre Vorstellung von »einer anderen Form des Sozialismus « nicht nur zu beschwören, sondern sie - wenn auch b eschränkt auf die ökonomische Ebene - zumindest als Zielvorstellung in den konkreten kubanischen Verhältnissen ein Stückweit zu füllen. Zentral ist für sie dabei die Unterscheidung zwischen Marktmechanismen und Besit zverhältnissen. So wird einem »regulierten Markt« (S. 151f) mittelfristig e ine zwar nicht absolute, aber doch weitgehende Steuerungsfunktion in der Ökonomie zugewiesen. Und sie sehen diese gebändigten Marktmechani smen keineswegs als nur notgedrungen zu akzeptierendes Übel und Zug eständnis an die schlechten Zeiten, sondern als integralen Bestandteil »eines normalen Funktionierens einer sozialistischen Wirtschaft« (S. 14), die aus dem Scheitern der sowjetischen und osteuropäischen Modelle gelernt hat:

»Oft wurde der Sozialismus für die erste der nicht mehr geldvermittelten Gesellschaften gehalten, dabei ist er in Wirklichkeit - im besten Falle - die letzte der geldvermittelten Gesellschaften« (S. 14).

Nicht die Negation von Marktmechanismen, sondern vielmehr »die Heg emonie des gesellschaftlichen Eigentums ist die conditio sine qua non eines sozialistischen Projekts« (S. 6). Dabei betonen die Autoren aber auch, daß es ihnen um die Hegemonie sozialen Eigentums geht, nicht um dessen Ver- 
absolutierung. Sie halten einen breiten (und durchaus zu fördernden!) Se ktor von wirklich unabhängigen, privaten Klein- und Mittelbetrieben (bis zu einer im einzelnen festzulegenden Größe) für unverzichtbar. Diese Priva tunternehmen sollen Läden und Lokale mieten und Lohnarbeiter anstellen dürfen - alles Punkte, die etwa im nach wie vor kommunistisch regierten Vietnam längst selbstverständlich sind, die aber über die in Kuba bisher zugelassene, vielfältig beschränkte »Ar beit auf eigene Rechnung« meilenweit hinausgehen.

Für große Bereiche der Wirtschaft sehen die Autoren in Genossenschaften adäquate Lösungen, um nicht-privates Eigentum mit ökonomischer Effiz ienz zu verbinden. Schlüsselbereiche der Wirtschaft sollten aber auch lan gfristig staatlich bleiben. Allerdings müßten auch die staatlichen Unterne hmen ein Korrektiv in Form von Marktmechanismen zu spüren bekommen und dürften nur in begründeten Fällen und in begrenztem Maße auf staatl iche Subventionen zählen. Wenn die Verfasser hier unter anderem ein Ko nkursgesetz vorschlagen, weil in letzter Instanz auch ein Staatsbetrieb keine Ewigkeitsgarantie ungeachtet seiner produktiven Leistung haben könne, dann geht auch dies sehr viel weiter als alles, was bisher in Kuba zum Thema Effizienzsteigerung des Staatssektors geäußert wurde.

Schon im Vorwort schreiben Carranza et al., daß sie sich des $»$ Risikos, für die Geschichtsbücher zu schreiben«, bewußt seien, »ein zwar löbliches Unterfangen, aber nicht Absicht dieses Buches« (S. VII). Zwischen dem Zeitpunkt seines Entwurfs - April 1994 - und dem seiner Veröffentlichung Herbst 1995 - wurde jedoch viel Zeit verloren und es wurden entscheide nde Weichen anders gestellt. Die Autoren haben verschiedene Versuche u nternommen, ihr Konzept den veränderten Voraussetzungen entsprechend zu aktualisieren. Spätestens aber mit der vernichtenden Brandrede Raúl Castros vor dem 5. Plenum des ZK über »die bittere Erfahrung, die man mit dem Zentrum für Amerika-Studien gemacht hat« (R. Castro 1996, 7), der Entlassung des Direktors des CEA und der Versetzung von Carranza und anderen CEA-Wissenschaftlern an andere Einrichtungen liest sich das Buch letztlich doch als eine Publikation »für die Geschichtsbücher«: als verpaßte Gelegenheit; als Kubas Reform, die nicht stattfand; als der nicht unternommene Versuch einer grundlegenden Transformation der kuban ischen Ökonomie von innen heraus und mit sozialistischem Vorzeichen.

\section{Das Problem der Demokratisierung in Kuba, oder: Versuche im Dunkeln über ein Minenfeld zu laufen}

Die Reaktion der Staatsführung - dies ist hier zu unterstreichen - richtete sich nicht allein gegen eine bestimmte einzelne Publikation oder Person. Die Attacke nahm zwar konkret das Zentrum für Amerika-Studien ins Visier, 
machte dieses allerdings zum exemplarischen Fall; der Angriff galt den k ubanischen Sozialwissenschaften insgesamt, die in einer Vielzahl von Bere ichen versuchten, über die bisherigen Beschränkungen hinauszukommen.

Überflüssig zu sagen, daß es sich dabei ausnahmslos um Diskussionen handelte, die sich innerhalb des aktuellen politischen Systems verorteten. Überflüssig auch, daß dieser neue Schwung nicht alle gleichermaßen betraf und ein großer Teil des akademischen Establishments recht unberührt d avon in alten Bahnen weiterarbeitete.

A propos: Wer daran Interesse hat, dem sei der Vergleich zwischen der A rbeit von Carranza et al. und der herkömmlichen Behandlung des Themas etwa in dem nur ein Jahr zuvor im gleichen Verlag und in gleichem U mfang erschienenen Buch von Miguel Alejandro Figueres (1994): Aspectos estructurales de la economía cubana (Strukturelle Aspekte der kubanischen Wirtschaft) empfohlen. Figueres präsentiert eine Fülle von Daten, Produktionszahlen und Tonnenerträgen mit flächendeckendem Optimi smus, allerdings keinerlei Analyse ökonomischer Funktionsmechanismen. Beispielhaft das Kapitel mit dem Titel: »Beschäftigung - Mangel oder Überfluß?« (S. 12f): in keiner Zeile geht der Autor auf die Frage ein, die er in der Überschrift formuliert, sondern fährt stattdessen materialreich die stolze Bilanz des hohen Ausbildungsstands und der Integration der Frau in das Arbeitsleben auf, ohne auch nur - 1994! - ein einziges Problem anz usprechen, nicht Unterbeschäftigung, nicht Arbeitslosigkeit, nicht die g esunkene Arbeitsproduktivität, nicht den Wertverlust der Löhne, nichts. Für derartige Wissenschaft gibt es keinen Ärger von oben, sondern Aufstieg: Figueres firmiert heute als »Berater des Ministers «.

Neben der Debatte um die wirtschaftlichen Reformmaßnahmen stand im Zentrum des Aufbruchs in Kubas Sozialwissenschaften die Suche nach e inem erneuerten Verständnis von Politik und Gesellschaft. Dabei ging es zum einen um neue Fragen, Themen und Begriffe: Dezentralisierung, Par tizipation, Ausdifferenzierung der Lebenswelten, Heterogenisierung der Bevölkerung, Zivilgesellschaft, Fragen der Emigration und der Beziehungen zu den Kubanern im Ausland wurden einige der leitmotivisch wiederkehrenden Stichworte. Zum anderen ging es aber auch um einen Wandel in der Art und Weise, in Kuba Sozialwissenschaft zu betreiben: Ein starkes B estreben, nach Jahrzehnten der Einbindung in die akademischen $Z$ usammenhänge und den Diskurs des real existierenden Sozialismus wieder ve rstärkt Anknüpfungspunkte an die Diskussionen in Lateinamerika, den USA und Europa zu finden; Ansätze zur Kontroverse mit ausländischen, auch nicht-sozialistischen Autoren in den eigenen Publikationen sowie eine pluraler und kontroverser geführte Debatte unter den kubanischen Wisse nschaftlern und Intellektuellen selbst; eine neue Nüchternheit bei der Anal yse der Probleme des Landes; und ein Selbstverständnis der Wissenschaftler 
eher als »Avantgarde im Fragen stellen und Probleme aufzeigen« denn als akademischer Resonanzkörper für die Politik der Exekutive.

Ein gutes Beispiel bietet der von Haroldo Dilla (1995), ebenfalls ein Wissenschaftler vom Zentrum für Amerika-Studien, herausgegebene Samme lband: La democracia en Cuba y el diferendo con los Estados Unidos (Die Demokratie in Kuba und der Konflikt mit den USA). Der Band vereint die Beiträge einer Konferenz, die das CEA im Mai 1994 veranstaltet hatte und zu dem auch eine Reihe ausländischer Wissenschaftler eingeladen waren. Seine Publikation wurde finanziell unterstützt durch die Grünen-nahe deutsche Stiftung »Buntstift« und den zentralamerikanischen Forschungsverbund CRIES. Unter den Autoren aus dem Ausland finden sich solch pr ominente Namen wie der Harvard-Professer Jorge Domínguez oder der ehemalige US-Diplomat Wayne Smith, der unter Jimmy Carter Leiter der Interessenvertretung der USA in Havanna war. Auf der Basis einer klaren Begrenzung des politischen Spektrums (alle Eingeladenen zählen in den USA zweifellos zu den $»$ Moderaten «) betont Dilla in der Einleitung die neue Toleranz: »Das Buch wird durch eine Verschiedenheit der Sichtwe isen gekennzeichnet, die alle mit vernünftigen Argumenten vorgetragen werden« (S. 6). Gleichzeitig grenzt sich der Herausgeber inhaltlich von den nicht-sozialistischen Autoren ab: » Smith und vor allem Domínguez liefern eine kritische Analyse, die von einer implizit liberal-demokratischen Grundhaltung ausgeht und die (...) eine Neudefinition des Systems bede uten könnte, die einer sozialistischen Zukunft entgegensteht« (S. 7). Was eher untertrieben ist: In einer klug argumentierenden Analyse mißt Domínguez das aktuelle kubanische System an dessen eigenen demokratischen Ansprüchen und kommt dabei zu in der Tat wenig erbaulichen Ergebni ssen; in der Folge läßt er keinen Zweifel daran, daß für ihn einzig ein Meh rparteiensystem und freie Wahlen echte Demokratie darstellen (S. 129). Dem stellen Dilla selbst und andere kubanische Autoren eine Reihe von Aufsätzen entgegen, die dem Status quo gegenüber zwar punktuelle Kritik äußern, aber grundsätzlich das bestehende System verteidigen.

Von den Hardlinern in Kuba haben Publikationen mit einer derartigen »Verschiedenheit der Sichtweisen« dennoch den Vorwurf erhalten, »Geg nern der Revolution « Raum in eigenen Publikationen zu geben. Der G edanke der kubanischen Wissenschaftler - und Dilla gehört mit Sicherheit eher zu den Kritikern »von links« als zu denen, die auf US-amerikanische Lösungen hoffen - war indes ein anderer, der sich ungefähr so zusamme nfassen läßt: Wenn die Position der kubanischen Revolution nur ohne G egenstimmen geäußert werden darf, dann mag sie zwar einen Sieg nach dem anderen erringen, nur werden diese Siege immer wertloser. Überzeugend werden kann die eigene Position erst, wenn sie auch neben anderen Me inungen Bestand hat - zumal in einer eigenen Publikation die Herausgeber 
die Spielregeln bestimmen und Manipulationen der $\gg$ Gegner der Revolut ion « damit ausgeschlossen sind.

Der eigene Beitrag von Haroldo Dilla beginnt mit dem Satz: »Über das Problem der Demokratie (und der Demokratisierung) der kubanischen G esellschaft zu schreiben, wie ich es mir hier vornehme, bedeutet, im Du nkeln über ein Minenfeld zu laufen« (S. 169). Die Instrumentalisierung des Demokratiebegriffs durch die US-Politik, auf die Dilla in der Folge verweist, ist in der Tat ein Hindernis allererster Ordnung für dessen unbefa ngene Diskussion in Kuba: Wer mehr Demokratie im Lande fordert, wird schnell zum Handlanger des Imperialismus erklärt, weil dieser ja auch i mmer »Demokratie« im Munde führe. Bei den kubanischen Beiträgen in dem Band bleibt der Leser daher allzuoft darauf angewiesen, zwischen den Ze ilen zu lesen oder den kritischen Gehalt aus den Nuancen herauszufiltern. Wer auf Zehenspitzen geht, der ist schwer zu hören. Vieles wird ausg eklammert - Fidel Castro etwa ist komplett tabu. Anderes erscheint ausg esprochen zaghaft, und die angedeuteten Veränderungen wirken angesichts der Probleme als viel zu kurz gegriffen. Im Nachhinein ahnt man, daß sie angesichts der Machtverhältnisse bereits »zu weit « gegangen waren.

Das Buch hat einen Nachfolger, gleichsam einen zweiten Teil: La participación en Cuba y los retos del futuro (Die Partizipation in Kuba und die Herausforderungen der Zukunft), ebenfalls herausgegeben von Haroldo Dilla (1996). Wo es zuvor um das politische System im engeren Sinne ging, werden hier die verschiedenen Bereiche der »Partizipation« auf g esellschaftlicher Ebene verhandelt, diesmal ausschließlich von kubanischen Autoren. $\gg$ Ein gemeinsamer Nenner der hier versammelten Arbeiten kann benannt werden«, schreibt Dilla in der Einleitung: »Die Idee, daß die kub anische Gesellschaft partizipativer sein sollte (...) und daß diese Partizipat ion gedacht werden muß als eine größere Autonomie der Einzelnen und der Organisationen《 (S. 8).

Niurka Pérez Rojas und Cary Torres Vila von der Universität Havanna stellen sich die Frage nach Partizipation und Autonomie am Beispiel der Landwirtschaft, in der 1993 der Großteil der bis dahin staatlichen Großb etriebe in neue Agrargenossenschaften umgewandelt wurden, die sog enannten »Basiseinheiten der Genossenschaftlichen Produktion« (UBPC). Wie die Autorinnen darstellen, handelte es sich dabei um »eine Transfo rmation von oben, die nicht auf Forderungen der Landarbeiter, sondern auf die tiefe Wirtschaftskrise reagiert《 (S. 169, Herv. im Original); Hauptg edanke war die Steigerung der wirtschaftlichen Effizienz, nicht eine Dem okratisierung der Arbeitsbeziehungen auf dem Land.

Durch die Analyse offizieller Dokumente, aber auch auf der Basis zahlre icher Interviews mit staatlichen Agrarfunktionären sowie Mitarbeitern der neuen Kooperativen belegen Pérez und Torres sehr konkret, wie sich der 
Staat ein so hohes Maß an Kontrolle über die neuen Genossenschaften vo rbehalten hat, daß deren formell erklärte Autonomie in der Praxis auf der Strecke bleibt. In der Folge fühlen sich die dort Arbeitenden kaum als Kooperativ-Bauern, sondern nach wie vor als Landarbeiter. »Der einzige U nterschied zur Staatsfarm ist, daß die UBPC ein eigenes Bankkonto hat «, $\mathrm{r}$ esümiert ein von Pérez/Torres interviewtes UBPC-Mitglied die aktuelle Situation.

Wenn die UBPC mehr als nur ein neues Etikett sein sollen, so die Botschaft der Autorinnen, dann muß den neuen Kooperativen auch reale Selbständi gkeit zugestanden werden. Wenn Pérez und Torres sich dabei am Ende vorsichtig optimistisch zeigen, dann ruht ihre Hoffnung weniger auf Erleuc htungen von oben als vielmehr auf den UBPC-Bauern selbst:

„In der Tat beginnt man eine gewisse Bewegung von unten zu erkennen, die zum einen den Widerstand der Mitglieder gegen die übermäßige Kontrolle und zum anderen das Verlangen nach einer Ausweitung ihrer Autonomie ausdrückt« (S. 182; Hervorhebung im Original).

Vor dem Hintergrund dieser Diskussionen über die wachsende Heterogenität der kubanischen Gesellschaft und deren notwendiger Autonomie vom Staat ist der Begriff der "Zivilgesellschaft« ins Zentrum gerückt. Dabei gewinnt die international oft diffuse Zivilgesellschaftsdiskussion in den konkreten kubanischen Verhältnissen eine eigene Dynamik. Angestoßen worden ist diese Debatte durch einen Aufsatz von Rafael Hernández, den Leiter der Nordamerika-Abteilung des CEA, zwei Jahre zuvor. In La participación en Cuba y los retos del futuro führt er seine Ửberlegungen weiter. Zivilgesellschaft definiert Hernández dabei keineswegs als Antithese oder in Opposition zum sozialistischen Staat, sondern als einen Bereich, der mit diesem in vielfältiger Weise in Beziehung steht. Und Hernández dreht den Spieß um: es ist Aufgabe des sozialistischen Staates, einerseits die Eige nständigkeit der Zivilgesellschaft zu respektieren und andererseits ihre Art ikulation mit dem politischen System zu organisieren:

»Als diejenige Sphäre, in der die Spannungen und Konflikte ausgetragen werden, die den Staat betreffen, muß es das Interesse und die Verantwortung des Staates sein, neue Quellen der Legitimation und Bereiche des Konsens in der Zivilgesellschaft zu suchen« (S. 88).

Rafael Hernández ist auch Leiter der seit Anfang 1995 erscheinenden Vierteljahresschrift Temas - Cultura, Ideologia, Sociedad, die sich bereits in ihren ersten Ausgaben als das zentrale interdisziplinäre Diskussionsf orum der kubanischen Sozialwissenschaften etabliert hat. Und wo in der Diskussion der letzten Jahre der Aufsatz zur dominierenden Publikation sform wurde (die Monographie von Carranza et al. ist die Ausnahme, die die Regel bestätigt), ist der Hinweis auf Temas unverzichtbar.

Im Editorial der ersten Ausgabe heißt es: »Temas entsteht inmitten der schwersten wirtschaftlichen und ideologischen Krise« - der offizielle Diskurs kennt nur erstere -, »die Kuba in den letzten Jahrzehnten erlebt hat« (S.3). Die 
Antwort, die Temas darauf gibt, sind zunächst einmal Fragen. Unter der Überschrift »Was denkt man in Kuba?« versucht der Themenschwerpunkt der Nummer Eins eine kritische Bestandsaufnahme der Gegenwart aus dem Blickwinkel der verschiedenen sozialwissenschaftlichen Disziplinen, von der Soziologie bis zur Psychologie, von der Geschichtsschreibung bis zur Phil osophie. Denn in vielen Bereichen kommt die Arbeit der letzten Jahre pra ktisch einer »zweiten Gründung« gleich - es ist daran zu erinnern, daß in den 70er Jahren etwa der Studiengang Soziologie an der Universität von Havanna komplett aufgelöst (und durch das Fach »Wissenschaftlicher Kommunismus « ersetzt) und erst Ende der 80er Jahre wiedereröffnet wurde; der ebenfalls A nfang der 70er Jahre abgeschaffte Fachbereich Politische Wissenschaft ist bis heute noch nicht wieder eing erichtet worden.

Die zweite Nummer von Temas setzt diese Bestandsaufnahme fort, indem sie weiterfragt: »Wie denkt man außerhalb Kubas über Kuba? « Eine Frage, die eine Antwort ist auf den immer wieder zu spürenden Hunger der kub anischen Wissenschaftler nach einer neuen Öffnung zur internationalen Wissenschaftsszene. Der Temas-Schwerpunkt umfaßt dabei sowohl Aufsätze »von außen «, etwa über den Stand der Cuban Studies in den USA, als auch Aufsätze von kubanischen Wissenschaftlern über die Wahrnehmung Kubas im Ausland.

Dabei führt der Blick nach außen auch zurück zu den Verhältnissen in $\mathrm{K}$ uba selbst, wenn etwa Ernesto Rodríguez Chávez vom Zentrum für Amerika-Studien in Havanna eine »kritische Bilanz der kubanischen Debatte über die 'Kubanologie'» zieht: »Kubanologie«, das bezeichnet im offiziellen Sprachgebrauch all jene ausländischen Arbeiten über Kuba, die nicht expl izit die Unterstützung der Politik der kubanischen Regierung zum Ziel h aben. Rodríguez Chávez argumentiert, es sei Zeit, auch in Kuba diesen pa uschalen pejorativen Terminus ad acta zu legen und die Auseinandersetzung mit den Kuba-Studien außerhalb der Insel sachlicher zu führen - ohne mit ihnen einer Meinung sein zu müssen. Trotz der richtigen Kritik an den allzuoft ideologisch voreingenommenen Arbeiten aus dem Ausland dürften diese nicht nur in den für die allgemeine Nutzung gesperrten Giftschränken der Biblioteca Nacional versauern: "Es ist nicht möglich, in der wisse nschaftlichen Erkenntnis über die kubanische Gesellschaft voranzukommen, ohne die Arbeiten derer zu kennen, die uns von außen mit sehr viel krit ischeren Augen sehen«, resümiert Rodríguez Chávez (S. 84). Die Antwort der Regierung auf diese Vorschläge sollte einige Monate später der Bericht des Politbüros geben; mit aller Entschiedenheit bekämpfen müsse die Pa rtei, so Raúl Castro explizit, einen jeden »kubanischen Wissenschaftler, der in Wirklichkeit zu einem Kubanologen mit kubanischer Staatsbürgerschaft - und teilweise sogar mit dem Parteibuch! - geworden ist und seine Positi onen mit der Zustimmung unserer Feinde verbreitet« (R. Castro 1996, 7). 
Als Raúl Castro diesen Bericht des Politbüros vor dem 5. Plenum des Zentralkomitees der kubanischen KP verlas, war gerade die vierte Ausgabe von Temas im Druck. Sie enthält neben einem ausführlichen Themenschwe rpunkt über die Religionen in Kuba einen bemerkenswerten Aufsatz von Hugo Azcuy (CEA) über »Staat und Zivilgesellschaft in Kuba«, der an die Überlegungen von Rafael Hernández anknüpft. Angesichts der Pluralisierung der Interessenlagen sei die kubanische Gesellschaft nicht mehr repr äsentativ in den sechs für alles zuständigen kommunistischen »Massen organisationen « zu organiseren; das Konzept der Zivilgesellschaft sei daher nicht nur als Instrument der Analyse zu gebrauchen, sondern vielmehr auch als ein gesellschaftliches Projekt zu denken, das mit einer Vielzahl von e igenständigen, nicht-staatlichen Organisationen der neuen Vielfalt der L ebenswirklichkeiten und Interessen in der kubanischen Gesellschaft Rec hnung tragen müßte - immer innerhalb des Rahmens der Revolution und der sozialistischen Ordnung, versteht sich.

Azcuy weist dabei selbst auf das Problem hin, daß der Begriff »Zivil gesellschaft« auch von Gegnern der Revolution und in der Propaganda der USPolitik gebraucht wird, die darin ein Einfallstor zum Sturz des Systems sehen. Dies sollte die Kubaner jedoch nicht daran hindern, so Azcuy, ihr eigenes Konzept einer zivilgesellschaftlichen Öffnung in und für den Sozialismus zu verfolgen. Das kubanische Denken dürfe sich nicht ex negativum von seinen Gegnern diktieren lassen - erst recht nicht bei einem Begriff, der auf eine s olide Verankerung auch in der marxistischen Tradition bauen kann.

Bei der Staatsführung stießen diese Argumente auf taube Ohren. Der Bericht des Politbüros liest sich vielmehr geradezu wie ein negatives Spiege 1bild des Artikels von Azcuy, wenn er über ganze Passagen hinweg die "Zivilgesellschaft《 als Machenschaft des Feindes geißelt und » NichtRegierungs-Organisationen« als » Trojanisches Pferd, das die Spaltung und die Subversion stimulieren soll« entlarvt.

Dabei waren die Vorwürfe in dem Bericht des Politbüros zwar denkbar massiv, aber auch komplett unpräzise: Keine einzige Publikation wurde beim Namen genannt, kein Wissenschaftler, kein Seminar. Lediglich das CEA wurde als Institution explizit erwähnt, und sein Direktor unmittelbar im Anschluß an die Rede vor dem Plenum des Zentralkomitees seines Amtes enthoben. Darüber hinaus wurden die Folgen für die vermeintlichen Parteigänger des imperialistischen Feindes in harten Worten angekündigt, aber im einzelnen gänzlich im Unklaren belassen. Und genau diese kalk ulierte Ungewißheit hat System. Gerade weil die Drohung so fulminant, aber völlig ungenau ist, wird die Einschüchterung so flächendeckend wirksam: Niemand weiß, wer etwas auf den Kopf bekommt, aber alle ducken sich. Auch ein Jahr nach der Rede Raúl Castros gibt es noch kein klares Bild über die Konsequenzen dieses »ideologischen Feldzuges«. Das intellekt u- 
elle Klima, so viel ist klar, ist frostiger geworden. Einen Kahlschlag auf breiter Front hat es allerdings nicht gegeben. In allen wissenschaftlichen Studienzentren wurden Evaluierungskommissionen eingesetzt, die die bisherige Arbeit im Lichte der Orientierungen des Politbüros überprüfen sol 1ten. Im Fall des Zentrums für Amerika-Studien war der Leiter dieser Kommission kein geringerer als José Ramón Balaguer, hochrangiges Politbüro-Mitglied und Chefideologie der KP. Der entlassene Direktor des CEA, Luis Suárez Salazar, präsentierte sich einige Zeit später in einer ö ffentlichen Selbstkritik als reuiger Sünder, der der Partei dafür dankte, wi eder auf den rechten Pfad zurückgeführt worden zu sein. Darüber hinaus wurden konkrete Ergebnisse dieser Evaluierungen nirgendwo öffentlich gemacht. Inoffiziell war zu hören, daß dem CEA die Zuständigkeit, über Kuba zu arbeiten, gänzlich entzogen werden soll, und es sich künftig allein dem Studium anderer Länder widmen solle. Zahlreiche prominente Mita rbeiter des Zentrums, darunter auch Carranza und Dilla, haben das CEA verlassen bzw. »wurden gegangen«. Gleichwohl haben sie keineswegs B erufsverbot erhalten, sondern arbeiten nun an der Universtität oder anderen akademischen Einrichtungen weiter, wenn auch, wie anzunehmen ist, unter engerer ideologischer Kontrolle.

Auch die Zeitschrift Temas existiert weiter, und die im Druck befindliche vierte Ausgabe erschien im Prinzip planmäßig als ob nichts geschehen sei. Kurz nach der Rede Raúl Castros und noch vor dem Erscheinen der Zei tschrift war jedoch Hugo Azcuy - der durch den Bericht des Politbüros direkt angegriffene Autor des Aufsatzes über das »Projekt Zivilgesellschaft« - an einem Herzinfarkt verstorben, so daß die öffentliche Präsentation der vierten Ausgabe in einer denkbar bedrückenden Atmosphäre verlief.

Für die Zukunft bleibt abzuwarten, inwieweit sich der von Raúl Castro verkündete Roll-back auf das inhaltliche Profil der Zeitschrift niederschlagen wird. Die inzwischen erschienene fünfte Ausgabe deutet auf keinen grun dsätzlichen Bruch, aber doch eine spürbare Zurückhaltung hin. Der Theme nschwerpunkt »Frauen in Kuba« entspricht ganz der bisherigen Linie, auch die Gesprächsrunde über »Globalisierung von links« mit kubanischen und ausländischen Wissenschaftlern. Die Zivilgesellschaftsdebatte allerdings wird mit einem Beitrag von Carlos Vilas fortgeführt, der vor allem den Mißbrauch dieses Konzepts in Lateinamerika durch rechte Kräfte b eschreibt. Und dann sticht am meisten ins Auge, was fehlt: ein Nachruf auf Azcuy; er wäre in einer derartigen Publikation in Kuba unbedingt zu e rwarten gewesen, geradezu »Pflicht«, schließlich war Azcuy langjähriger Wissenschaftler am Zentrum für Amerika-Studien und damit direkter Kollege von Temas-Herausgeber Hernández, aktives Mitglied auch der Parte izelle im CEA und einer der prominenten Mitarbeiter der Zeitschrift. 
Unterdessen haben die Hardliner in der Parteiführung nun eine eigene $\mathrm{P} u-$ blikation aus der Versenkung geholt, die ganz offenkundig Richtlinie nfunktion für den künftigen akademisch-politischen Diskurs haben soll: Die einst eingestellte Cuba Socialista ist wieder neu aufgelegt worden. Als Herausgeber firmiert das Zentralkomitee der Kommunistischen Partei K ubas, Präsident des Redaktionsrats ist just der Leiter der Evaluierungskommission im CEA und Chefideologie der KP, José Ramón Balaguer. Gleich im Editorial der ersten Ausgabe wiederholt Cuba Socialista die Grundlinien des Berichts des Politbüros:

»Einem Gedankengut, das unseren Zielen fremd ist, oder byzantinischen Streitereien werden wir keinen Platz bieten (...) Unsere Einbindung in die internationale Ökonomie und die Inv estitionen von ausländischem Kapital werden nie eine Öffnung für eine 'ideologische Invasion' darstellen, die unseren von Martí und von Marx geprägten Prinzipien fremd oder entgegeng esetzt ist. (...) Diejenigen, die - auch wenn sie es nicht einsehen wollen - die Fahnen der Rev olution und des Sozialismus eingerollt haben, werden hier keinen Raum finden« (S. 3f).

Wenn es im gleichen Editorial heißt, daß die Zeitschrift »nicht die offizie 1len 10 Gebote aufstellen soll, vor denen sich alle zu unterwerfen haben« (ebenda), dann ist dies angesichts der Umstände ihres Erscheinens und der prominenten Herausgeberschaft ein klassischer Fall jener Dementis, bei denen just das Dementierte die eigentliche Botschaft ist.

Der Inhalt der ersten Ausgabe von Cuba Socialista übertrifft noch die Erwartungen, die das Editorial weckt. Zum einen ist es die Rückkehr zu einer komplett affirmativen akademischen Arbeit, die sich, wie es ein Aufsatz des Philosophie-Professors Limia David in Temas No 1 selbstkritisch für die überwunden geglaubte Vergangenheit beschrieb, lediglich »als andere Daseinsform des offiziellen politischen Diskurses« (S. 21) versteht. Die Herausgeber von Cuba Socialista würden dies vermutlich nicht als Kritik sehen: Den zentralen ersten Aufsatz über »Kuba und den Kampf für die Demokratie in der Welt von heute«, schreibt Ricardo Alarcón, seines Zeichens Präsident des kubanischen Parlaments, den folgenden Grundsatzart ikel über »Kuba 1990-1995: Überlegungen zu einer richtigen Wirtschaft spolitik « der Wirtschaftsminister José Luis Rodríguez.

Erschreckender noch als diese Rezentralisierung des akademischen Disku rses ist seine »Caudillisierung «, wie sie Cuba Socialista als Leitlinie vorgibt. Fidel ist überall. Als Titelfoto Fidel in jungen Jahren und in kämpfer ischer Pose, Zitate von Fidel vor dem Editorial, Zitate von Fidel im Editor ial, das ganze Heft hindurch Zitate von Fidel und noch mehr Zitate von F idel in Kästen vor, nach und mitten in den Texten. Und wenn der Aufsatz von José Luis Rodríguez - vor Antritt seines Ministeramts vor drei Jahren einer der angesehensten Ökonomen Kubas - mit den Worten endet: »... g etreu den Ideen von Marx und Lenin, Martí und Fidel« (S. 28), dann ist die Botschaft deutlich: Cuba Fidel-ista. 
Es wird abzuwarten sein, was sich aus dieser Konfliktsituation für die da rgestellte Renaissance der kubanischen Sozialwissenschaften ergibt. Zum einen war die Reaktion der Parteiführung auf die Pluralisierung des Diskurses unmißverständlich feindlich - auch wenn der große Knüppel, der g eschwungen wurde, letztlich mehr als Drohung über den Köpfen blieb als daß er tatsächlich auf die Angeklagten niederfuhr. Andererseits ist aber auch nicht vorstellbar, daß der intellektuelle Aufbruch der letzten Jahre sich per ordre de mufti beenden und sich die Köpfe der kubanischen Wisse nschaftler auf Dauer zurück in die 70er Jahre drehen lassen. »Was einmal gedacht wurde«, schrieb Dürrenmatt in Die Physiker »kann nicht mehr zurückgenommen werden.« Vielleicht läßt sich dies ja auch optimistisch de nken.

\section{Literatur}

Carranza, Julio (1992): Cuba. Los retos de la economía; in: Cuadernos de Nuestra América, Vol. IX, No. 19, La Habana: Centro de Estudios sobre América, jul-dic 1992 (erschienen 1993); 131-159. Auf deutsch in: Hoffmann 1994, 16-41.

Carranza, Julio; Gutiérrez, Luis; Monreal, Pedro (1995): La restructuración de la economía cubana. Una propuesta para el debate, La Habana: Editorial Ciencias Sociales (1997 auch bei Editorial Nueva Sociedad, Carracas; engl.: Cuba: Restructuring the Economy - A contribution of Latin American Studies, University of London 1996).

Castro, Raúl (1996): Informe del Buró Politico (vor dem V. Plenum des Zentralkomitees der Kommunistischen Partei Kubas am 23.3.1996), in: Granma Internacional, 10.4.96, 4-8, La Habana.(Dokumentiert auch in: Spiegel der lateinamerikanischen Presse 3/96 des Instituts für Iberoamerika-Kunde Hamburg.)

Cuba Socialista (1996); 3ra época, Nr. 1, La Habana (vierteljährlich erscheinende Zeitschrift, herausgegeben vom Zentralkomitee der Kommunistischen Partei Kubas).

Dilla, Haroldo (Hg.) (1995): La democracia en Cuba y el diferendo con Los Estados Unidos; La Habana: Ediciones CEA (Centro de Estudios sobre América).

Dilla, Haroldo (Hg.) (1996): La participación en Cuba y los retos del futuro; La Habana: Ediciones CEA (Centro de Estudios sobre América).

Figueres, Miguel Alejandro (1994): Aspectos estructurales de la economia cubana, La Habana: Editorial Ciencias Sociales.

Hoffmann, Bert (Hg.) (1994/1996a): Wirtschaftsreformen in Kuba. Konturen einer Debatte, Frankfurt/M.: Vervuert 1994 (2. akt. Auflage 1996).

Hoffnann, Bert (1996b): Kubanische Comebacks - Die Rückkehr der Vergangenheit im sozialistischen Kuba; in: Gabbert et al: Offene Rechnungen - Lateinamerika Analysen und Berichte 20, Bad Honnef: Horlemann.

Marquetti, Hiram (1995): La liberalización de la circulación de divisa en Cuba-Resultados y Problemas (Manuskript); La Habana: Centro de Estudios sobre la Economía Cubana (CEEC), Oktober 1995, $22 \mathrm{~S}$.

TEMAS - Cultura, Ideologia, Sociedad (Nueva época), La Habana; vierteljährlich erscheine nde Zeitschrift: Nr. 1: Jan-März 1995; Nr. 2: Apr-Juni 1995; Nr. 3: Juli-Sep 1996; Nr. 4: Okt-Dez 1995. 